\title{
A game theory model based on Gale-Shapley for dual-resource constrained (DRC) flexible job shop scheduling
}

\author{
Paolo Renna ${ }^{a^{*}}$, Matthias Thürer ${ }^{\mathrm{b}}$ and Mark Stevenson ${ }^{\mathrm{c}}$
}

${ }^{a}$ School of Engineering, Università degli Studi della Basilicata, Italy

${ }^{b}$ Institute of Physical Internet, School of Electrical and Information Engineering, Jinan University (Zhuhai Campus), 519070, Zhuhai, PR China

${ }^{c}$ Department of Management Science, Lancaster University Management School, Lancaster University, LA1 4YX, United Kingdom

\section{H R O N I C L E}

\section{Article history:}

Received July 162019

Received in Revised Format

October 292019

Accepted October 292019

Available online

November 12019

Keywords:

Dual-resource constrained (DRC)

shops

Flexible job shop scheduling

Game theory

Gale-Shapley

Simulation \begin{abstract}
A B S T R A C T
Most job shops in practice are constrained by both machine and labor availability. Worker assignment in these so-called Dual Resource Constrained (DRC) job shops is typically solved in the literature via the use of meta-heuristics, i.e. "when" and "where" rules, or heuristic assignment rules. While the former does not necessarily lead to optimal results, the latter suffers from high computational time and complexity, especially when there is a large number of workstations. This paper uses game theory to propose a new worker assignment rule for DRC job shops. The Gale-Shapley model (also known as the stable marriage problem) forms a 'couple' made up of a worker and machine following a periodic review strategy. Simulation is used to evaluate and compare the proposed model to "when" and "where" rules previously proposed in the literature. Simulation experiments under different conditions demonstrate that the Gale-Shapley model provides better results for worker assignments in complex DRC systems, particularly when the workers have different efficiency levels. The implications of the findings for research and practice are outlined.
\end{abstract}

\section{Introduction}

In recent years, the rapid introduction of new products with shorter life cycles have required shops to adapt to rapid changes in the product mix and have driven firms towards adopting more flexible and customized job shop manufacturing systems. Job shop manufacturing systems have been widely investigated in the extant literature, but the majority of this research has considered only a single capacity constraint, i.e. based on the availability of machines only. In practice however, these manufacturing systems are typically constrained by two elements, i.e. by both machines and workers (Patel, 1997). These so-called Dual Resource Constrained (DRC) systems have received limited attention in the literature, where a job can only be processed if both a machine and a skilled worker is available. The DRC shop modelled in the literature is characterized by the number of workers rather than the number of machines, with the number of machines typically considered fixed. Since the number of workers is normally less than the number of machines, worker reallocations amongst the machines are necessary.

* Corresponding author

E-mail: paolo.renna@unibas.it (P. Renna)

2020 Growing Science Ltd.

doi: $10.5267 /$ j.ijiec.2019.11.001 
In such a context, the assignment of workers to machines becomes highly relevant when searching for improvements to manufacturing system performance. The job shop environment is not the only context in which dual resource constraints occur. Hence, the DRC problem has been modelled in several other applications, such as in hybrid push-pull control with a kanban system (Salum and Uraz, 2009), in cellular manufacturing systems (Kannan \& Jensen, 2004), in virtual cellular manufacturing systems (Hamedi et al., 2012), and automobile assembly plants (Fredendall et al., 1996), in addition to call centers, field service systems, serial production systems, manufacturing cells, etc. (e.g. Hopp \& Van Oyen, 2004; Nembhard, 2007). In this study, we focus on the job shop given the importance of short-term worker assignment decisions in this context (Delgoshaei et al., 2017). The job shop scheduling problem is an $N p$-hard combinatorial optimization problem (Lenstra \& Rinnooy Kan, 1979). The DRC system is even more difficult to solve than the job shop problem because of the presence of two resource constraints. It therefore becomes difficult to use analytical approaches and there are high computational costs. As a consequence, the use of meta-heuristic methods has been widely proposed in the literature (El Maraghy et al., 2000; Li et al., 2016; Li \& Lang, 2011). The scheduling problem in DRC systems is resolved via "when" and "where" rules; the when rule determines the timing of when a worker is considered for transferal between machines while the where rule determines the machine to which the worker should be transferred (Bokhorst et al., 2004). Often however the meta-heuristic is unable to find a near optimum solution ( $\mathrm{Li}$ et al., 2016). There is thus a trade-off between computational time and near optimality.

In response to the aforementioned trade-off, this study proposes and evaluates the use of game theory for assignment problems in DRC shops. More specifically, we argue that the assignment problem of workers to machines can be adapted to the so-called 'stable marriage problem' studied by Gale and Shapley (1962). The problem is based on considering $n$ men and $m$ women, where each person has ranked all members of the opposite gender in order of preference. The preference of the persons is hereby translated to the characteristics of the workers (as efficiency) and machines (as the parts in the queue). The "when" rule follows a periodic review strategy while the "where" rule is based on the Gale-Shapley algorithm (Gale \& Shapley, 1962). The algorithm is adapted to the asymmetry of the number of workers and machines and appropriate preference functions are defined. The Gale-Shapley algorithm allows the time to take the decision and to obtain a suitable configuration to be reduced, including in the context of largesized problems. We therefore suggest that it could enable the computational complexity of the DRC problem to be reduced and that it could be applied to various DRC systems, including the DRC job shop. We use simulation to assess its performance compared with the meta-heuristics most typically applied in the literature.

The remainder of this paper is organized as follows. Section 2 discusses the literature on worker assignments in DRC systems and outlines the research questions that motivate our study. Section 3 describes the manufacturing system context investigated and the proposed model, based on game theory. The simulation model used to evaluate performance is then described in Section 4 before the results are presented in Section 5. Finally, conclusions, managerial implications and future research directions are provided in Section 6.

\section{Literature Review}

Most of the contributions presented in the extant literature on worker assignments in DRC shops have focused on "when" and "where" rules and proposed meta-heuristic and heuristic approaches. Some examples studied the production planning problem in DRC manufacturing systems (El Maraghy, 2000) using Genetic Algorithm (GA) with different algorithms to improve the solution as grey simulation technology and non-dominated sorting GA II (Li 2011), branch population to accumulate and transfer the evolutionary experience of parent chromosomes via pheromone (Li et al., 2006). The advantage of GA lies in its global optimization ability while the disadvantage of GA is its low local search ability and the time it takes to obtain the solution following several iterations. Araz and Salum (2010) proposed a real-time scheduling approach to select a predetermined scheduling rule in DRC manufacturing systems. 
The model needs the combination of Artificial Neural Networks, fuzzy inference system and simulation to provide the knowledge base. The main limitations of this methodology are the predetermination of the rules and the accuracy of the Artificial Neural Network model. Several heuristic models have also been proposed, such as the variable neighborhood search approach by Deming and Guo (2014). Meanwhile, Mehravaran and Logendran (2013) developed a linear mixed-integer mathematical model and three metaheuristic algorithms to solve a bi-criteria non-permutation flow shop with dual resources and sequencedependent setup times. Zhang et al. (2017) proposed a hybrid discrete particle swarm optimization algorithm to solve the DRC job shop scheduling problem with resource flexibility. The computation time is a critical issue that limits the application of this method. Zheng and Wang (2016) proposed a knowledge-guided fruit fly optimization algorithm with a new encoding scheme to solve the DRC flexible job shop problem with a makespan minimization criterion. Meanwhile, Sammarco et al. (2014) used a simulation based on agent system modelling to study the effect of several factors (i.e. workforce, distance, buffer capacity, when rule, and where rule) on performance measured in terms of work-inprocess (WIP), flow time, and production rates. The introduction of DRC in cellular manufacturing systems was studied by Satoglu and Suresh (2009) for the design of hybrid cellular manufacturing systems and in Hamedi et al. (2012) for virtual cellular manufacturing systems. They proposed mathematical models to design the cellular manufacturing systems, including the labor assignment problem. However, these models were static because they considered the design problem only. Finally, some models have been developed to be applicable in large-scale contexts or to make scheduling decisions in real-time. For example, Lobo et al. (2013a, 2013b) studied the assignment of each worker to a specific machine group in a DRC job shop to minimize the maximum lateness of jobs. Lobo et al. (2014) then revised the approach of Lobo et al. (2013b) for the application of large-scale problems with tractable computational complexity.

\subsection{Assessment of the Literature}

$\mathrm{Xu}$ et al. (2011) provided an overview of prior work on DRC systems and discussed some possible approaches and future directions for solving the resource scheduling problem in a DRC system. The authors argued that improved performance could be achieved via the use of a dynamic control system that is able to find, in real-time, the best dispatching rule to use based on the current shop state and aggregate performance for chosen performance measures (Araz, 2005, 2007; Araz \& Salum, 2008). Nevertheless, the meta-heuristic methods are also more flexible than simple heuristic assignment rules in terms of scheduling ability, which makes future research in this area relevant. The main limits of the literature on DRC systems concern the use of meta-heuristic or heuristic approaches with high computational complexity, especially when the larger scale characteristics of real industrial cases are considered. The mathematical models provided in the literature can be used in a specific context, but they cannot be adapted to different industrial cases. Yet, it is also argued that the simple "where" and "when" rules are unlikely to find near optimal solutions.

In response, we propose game theory as a novel approach for solving the worker assignment problem in DRC job shops. Game theory approaches are widely used in the literature to solve scheduling problems in different contexts, such as the parallel machine scheduling problem (Elisha et al., 2008; Opiyo et al., 2009), Buscemi et al. (2012) addressed the job scheduling problem in heterogeneous computational grids by exploiting the concept of Nash equilibrium, Li et al. (2012) presented integrated process planning and scheduling in a manufacturing system and Sun et al. (2014) applied a non-cooperative game theory with complete information to solve a flexible job shop problem. Meanwhile, Renna (2017) proposed a model to support reconfiguration activities in job shop systems based on the Gale-Shapley model. The proposed model based on game theory is solved in one step to take the decision and using limited information and lower computational complexity. However, despite their promise, game theory models have not been used to solve the worker assignment problem in DRC job shops. The Gale-Shapley Algorithm is computationally easy to manipulate (Teo et al., 2011) and suitable for short-term decision; Therefore, the first research question is: 
RQ1: Can the Gale-Shapley Algorithm be adapted to the DRC job shop problem under different numbers of workers, workers' efficiencies and inter-arrival times?

The second research question then asks:

RQ2: $\quad$ What is the performance improvement enabled by the Gale-Shapley Algorithm compared with simple when and where rules?

Our first research question will be answered in the next section where the Gale-Shapley algorithm is introduced. Then, a simulation model is developed to answer the second research question. The performance of the proposed model is compared to classical "when" and "where" rules considering different numbers of workers, worker efficiencies, and inter-arrival times.

\section{Research context and Game model}

The model considered is a randomly routed job shop (Conway et al., 1967) or pure job shop (Melnyk \& Ragatz, 1989; Oosterman et al., 2000). The manufacturing system consists of six workstations, and each workstation consists of one machine. Each job is assigned a random routing sequence, the processing time for each machine and the due date. The routing sequences assigned to jobs have an undirected flow. The assumptions of the manufacturing system are as follows: operations cannot be pre-empted; each machine can process only one task at a time; and, the queues are managed by the Earliest Due Date (EDD) policy to improve lateness performance. In this research, the material handling time is included in the machining time, and the handling resources are always available. The manufacturing system is characterized by one bottleneck, as described in Section 4.

\section{The notation is the following:}

$m=1, \ldots, M$ is the index of the machines or workstations (the terms can be interchangeable);

$i$ is the index of the generic part that enters the manufacturing system

$a_{i m}=1$ if the part $i$ requires the operation of the machine $m, 0$ otherwise;

Topim $_{\text {im }}$ is the process time of the part $i$ in the machine $m$

$W L_{m}$ is the workload of the machine/work center $m$;

queuem is the processing time of the parts in the queue of the machine/work center m;

$W L_{a v}$ is the average workload over all machines/workstations;

$w=1, \ldots, W$ is the index of the workers' set;

$U_{w, m}$ is the preference (Gale-Shapley model) of the worker $w$ for the $m$ machine;

$U_{m, w}$ is the preference (Gale-Shapley model) of the machine $m$ for the $w$ worker;

Eff $_{\mathrm{w}, \mathrm{m}}$ is the efficiency of the worker $w$ on the machine $m$;

$T p$ is the period time fixed to evaluate the reconfiguration of the machines.

\subsection{Gale Shapley Algorithm (Renna, 2017)}

This Section summarizes briefly the Gale- Shapley as reported also in Renna (2017). The Gale-Shapley algorithm (Gale \& Shapley, 1962) was developed to solve the stable matching problem in order to find a stable match between two equal sets of elements, given an ordering preference for each element. The algorithm defined two finite sets of players called the set of men (m) and set of women (w). Every member of each set has preferences over the members of the opposite sex. At the start of the algorithm, each $m$ and $w$ are free; the algorithm starts from the first $m$ that chooses the most preferred $w$. If the woman is free, then $w$ and $m$ become 'engaged'. If the $w$ is engaged to another $m$ ', the $w$ rejects the man ( $m$ or $m$ ') with the lower preference and becomes engaged to the man ( $m$ or $m$ ') with the higher preference. Then, the rejected man ( $\mathrm{m}$ or $\mathrm{m}$ ') is free and proposes to their subsequent preferred women. The algorithm is iterated until all men are engaged, the engaged pairs form the male optimal stable matching set. This algorithm needs a number of steps that, in the worst case, is quadratic in $n$ (that is, the number of men), and it guarantees that if the number of men and women coincide and all participants express a strict order over all the members of the other group, everyone gets married, and the returned matching is stable. A pseudo-code that briefly explains the algorithm is the following: 
GALE-SHAPLEY (Set M, Set W)

while there is an unmarried man, $m$ do

$m$ chooses the first woman $w$ on his preference list that he has not proposed to yet and proposes to her

if $w$ is unmarried or prefers $m$ over her current partner $m$ ' then

$w$ divorces $m^{\prime}$ and $w$ marries $m$

\subsection{The Gale-Shapley Model}

The approach proposed supports the allocation of workers to machines following a periodic review strategy. The machines and the workers need to be divided into men and women according to the GaleShapley algorithm: the women are workers and the men are the machines. Then, the function to compute the preference among the members of the two sets will be defined. When a job enters the manufacturing system, the routing $\left(a_{i m}\right)$ and process time $\left(\right.$ Top $\left._{i m}\right)$ is assigned to the part as described in the simulation model section below. Then, the workload of the machines is updated, as shown in Eq. (1):

$$
W L_{m}=W L_{m}+\sum_{m=1}^{M} T o p_{i m} * a_{i m}
$$

The workload of the machine $m$ is reduced by the Topim when the part $i$ leaves the machine $m$ after the process ends. The preferences of the women (workers) are computed as shown in Eq. (2) as follows:

$$
U_{w, m}=\alpha \cdot \text { queue }_{m}+\beta \cdot W L_{m}
$$

where, $\alpha$ and $\beta$ are the weight comprises $(0,1)$ and $\alpha+\beta=1$. The preference of the workers is higher for the machine with the most urgent job in the queue and highest workload. The workload considers jobs at other machines that will visit the machine $m$. The preferences of the men are the efficiency of the workers, as shown in Eq. (3):

$$
U_{m, w}=e f f_{w, m}
$$

If the workers have the same efficiency, the preference for the machines is the same.

\subsection{Benchmark Model}

The benchmark model to compare the proposed approach is based on a decentralized "when" rule because the Gale-Shapley algorithm can operate only as a decentralized rule. In other words, all operations at a station have to be completed before a worker can be reallocated. While this rule is outperformed by decentralized rules (Thurer et al., 2018) if worker transfer costs or delays are not considered, it leads to substantially lower worker movements or reassignments. This makes it more suitable for many practical situations. The "where" rule is based on the number of parts in the queue of the machines. Only one rule is considered since there were no significant performance differences between rules in Thurer et al. (2018).

\section{Simulation Model}

To evaluate the proposed model, when compared to the benchmark ("when"-"where" rules), the following simulation model is developed. Table 1 reports the characteristics of the models tested (Thurer et al., 2014a; Thurer et al, 2014b, Renna, 2015). The job shop consists of six workstations, and each work center is characterized by one machine. A random routing sequence without any directed flow is assigned to a job when it enters the manufacturing system. Then, the processing time and due date is assigned, as shown in Table 1. The main assumptions of the manufacturing system modelled (as in previous studies) are as follows: raw materials are always available; all jobs are accepted; operations cannot be pre-empted; each machine can only process one task at a time; the queues are managed by the EDD rule (to improve lateness performance); the material handling time is included in the machining time; and, the handling resources are always available. The inter-arrival parameter is adapted to the number of workers available; 
5 workers is the base; the other inter-arrival parameters are calculated by multiplying the base interarrival by (5/4) for the scenario with 4 workers and (5/3) for the scenario with 3 workers. All the models are tested with $T p$ periodic review from 1 to 17 and only the best case is reported in the numerical results. Four cases of workers' efficiency are considered. In the first case, all the workers have the same efficiency, then it is considered a difference of efficiency of $10 \%, 20 \%$ and $30 \%$ among the workers. Several values of $\alpha$ and $\beta$ are tested and only the best cases are reported in the numerical results.

Table 1

Model Characteristics

\begin{tabular}{|c|c|}
\hline Number of machines & $6(1$ bottleneck and 5 no bottlenecks $)$ \\
\hline Inter-arrival & $\begin{array}{l}\text { EXPO: } 0.7704(\mathrm{H}) ; 0.642(\mathrm{M}) ; 0.6099(\mathrm{~L}) \text { with } 5 \text { workers available } \\
\text { EXPO: } 0.963(\mathrm{H}) ; 0.8346(\mathrm{M}) ; 0.7704(\mathrm{~L}) \text { with } 4 \text { workers available } \\
\text { EXPO: } 1.284(\mathrm{H}) ; 1.156(\mathrm{M}) ; 1.0914(\mathrm{~L}) \text { with } 3 \text { workers available }\end{array}$ \\
\hline $\begin{array}{l}\text { Number of Operations } \\
\text { Due Date }\end{array}$ & $\begin{array}{l}\text { Discrete Uniform }[1,6] \\
\text { (Number of Operations)*(Total processing time)*Uniform }[5,10]\end{array}$ \\
\hline $\begin{array}{l}\text { Processing time } \\
\text { no bottlenecks }\end{array}$ & 2-Erlang with mean 1 \\
\hline $\begin{array}{c}\text { Processing time bottlenecks } \\
\text { Tp }\end{array}$ & $\begin{array}{l}\text { 2-Erlang with mean } 1.15 \text { (utilization about 90\%) } \\
1,3,5,7,10,12,15,17\end{array}$ \\
\hline Workers' efficiency & $\begin{array}{l}\text { a)Efficiency all equals (EFF 1); } \\
\text { b) efficiency by uniform distribution [0.9-1.1] (EFF 2); } \\
\text { c) efficiency by uniform distribution [0.8-1.2] (EFF 3); } \\
\text { d) efficiency by uniform distribution [0.7-1.3] (EFF4). }\end{array}$ \\
\hline
\end{tabular}

The performance measures concern the following:

- The ability to deliver the job on time is measured by the following indexes: the percentage of tardy jobs; the standard deviation of lateness; and, the average lateness [unit time]. The standard deviation of job lateness is a measure of how spread out a lateness distribution is. It is used as an indicator of timing performance, i.e. it indicates how close the jobs are completed to their due dates.

The manufacturing system is evaluated based on:

- Throughput [parts/unit time]; the total number of items processed/produced by the manufacturing system over the simulation time period.

- Average job-shop time [unit time]; it is the average time from the release time of the order in the manufacturing system to the time the order exits the system.

- Work In Process (WIP) [jobs]; it is the average total number of jobs in the system (the sum of the queues and jobs in the machines);

- Average Workload of the machines [unit time]; it is the average workload of a machine over the simulation period;

- Standard Deviation of the Workload of the machines [unit time]; it measures the distribution of the workload of the machines. This performance is reported as the coefficient of variation.

The performance about the workload should be relevant to compare this research to approaches that include also the workload control of the manufacturing system. The simulation length is 25,000 time units. For each experimental scenario, a number of replications have been conducted that are able to assure a $5 \%$ confidence interval and $95 \%$ confidence level for each performance measure. Each combination of the experiment class is characterized by over 3,000 replications and about 12 hours of computation time (4 GHz Intel Core i7 and $16 \mathrm{~Gb}$ RAM).

\section{Results}

Table 2 reports the Analysis of Variance (ANOVA) for the lateness performance (average, standard deviations- DS and percentage of items) and Table 3 reports the Analysis of Variance (ANOVA) for the system time, throughput, Work In Process (WIP), and Workload performance (average and standard deviation - DS). 
Table 2

ANOVA Results - Delivery Performance

\begin{tabular}{|c|c|c|c|c|c|c|}
\hline & Source of variance & $\begin{array}{l}\text { Sum of } \\
\text { Square }\end{array}$ & $\begin{array}{l}\text { Degree of } \\
\text { Freedom }\end{array}$ & Mean Square & F-value & P-value \\
\hline \multirow{8}{*}{ Lateness } & Efficiency & 6050.000 & 3 & 2016.700 & 14.420 & $\underline{0.000}$ \\
\hline & Inter-arrival & 4418.000 & 2 & 2209.000 & 15.790 & 0.000 \\
\hline & Workers & 1303.000 & 2 & 651.600 & 4.660 & $\overline{0.032}$ \\
\hline & Efficiency*inter-arrival & 5064.000 & 6 & 844.000 & 6.030 & $\overline{0.034}$ \\
\hline & Efficiency* Workers & 1338.000 & 6 & 223.000 & 1.590 & $\overline{0.231}$ \\
\hline & Workers* inter-arrival & 1749.000 & 4 & 437.300 & 3.130 & 0.056 \\
\hline & Error & 1678.000 & 12 & 139.900 & & \\
\hline & Total & 21601.000 & 35 & & & \\
\hline \multirow{8}{*}{ Lateness DS } & Efficiency & 1899.800 & 3 & 633.300 & 8.470 & $\underline{0.003}$ \\
\hline & Inter-arrival & 1834.900 & 2 & 917.500 & 12.260 & $\underline{0.001}$ \\
\hline & Workers & 674.600 & 2 & 337.300 & 4.510 & $\underline{0.035}$ \\
\hline & Efficiency*inter-arrival & 1107.100 & 6 & 184.520 & 2.470 & $\overline{0.086}$ \\
\hline & Efficiency* Workers & 724.700 & 6 & 120.760 & 1.610 & 0.226 \\
\hline & Workers* inter-arrival & 1029.100 & 4 & 257.280 & 3.440 & $\underline{0.043}$ \\
\hline & Error & 897.700 & 12 & 74.810 & & \\
\hline & Total & 8167.900 & 35 & & & \\
\hline \multirow{8}{*}{ Lateness $\%$} & Efficiency & 2.886 & 3 & 0.962 & 112.260 & $\underline{0.000}$ \\
\hline & Inter-arrival & 1.123 & 2 & 0.563 & 65.740 & $\overline{0.000}$ \\
\hline & Workers & 0.259 & 2 & 0.129 & 15.110 & $\overline{0.001}$ \\
\hline & Efficiency*inter-arrival & 0.433 & 6 & 0.072 & 8.420 & $\overline{0.001}$ \\
\hline & Efficiency* Workers & 0.233 & 6 & 0.039 & 4.540 & $\overline{0.013}$ \\
\hline & Workers* inter-arrival & 0.094 & 4 & 0.024 & 2.750 & $\overline{0.078}$ \\
\hline & Error & 0.103 & 12 & 0.009 & & \\
\hline & Total & 5.133 & 35 & & & \\
\hline
\end{tabular}

Table 3

ANOVA Results - Manufacturing System Performance

\begin{tabular}{|c|c|c|c|c|c|c|}
\hline & Source of variance & $\begin{array}{l}\text { Sum of } \\
\text { Square }\end{array}$ & $\begin{array}{l}\text { Degree of } \\
\text { Freedom }\end{array}$ & Mean Square & F-value & P-value \\
\hline \multirow{8}{*}{ System Time } & Efficiency & 9700.000 & 3 & 3233.300 & 21.830 & 0.000 \\
\hline & Inter-arrival & 5905.000 & 2 & 2952.300 & 19.930 & 0.000 \\
\hline & Workers & 1156.000 & 2 & 578.100 & 3.900 & $\overline{0.049}$ \\
\hline & Efficiency*inter-arrival & 5220.000 & 6 & 869.900 & 5.870 & 0.005 \\
\hline & Efficiency* Workers & 2002.000 & 6 & 333.700 & 2.250 & 0.109 \\
\hline & Workers* inter-arrival & 2028.000 & 4 & 506.900 & 3.420 & $\underline{0.044}$ \\
\hline & Error & 1778.000 & 12 & 148.100 & & \\
\hline & Total & 27788.000 & 35 & & & \\
\hline \multirow{8}{*}{ Throughput } & Efficiency & 0.000 & 3 & 0.000 & 0.500 & 0.687 \\
\hline & Inter-arrival & 0.376 & 2 & 0.188 & 112369.000 & $\underline{0.000}$ \\
\hline & Workers & 2.485 & 2 & 1.242 & 742144.000 & $\underline{0.000}$ \\
\hline & Efficiency*inter-arrival & 0.000 & 6 & 0.000 & 1.010 & $\overline{0.463}$ \\
\hline & Efficiency* Workers & 0.000 & 6 & 0.000 & 2.230 & 0.112 \\
\hline & Workers* inter-arrival & 0.050 & 4 & 0.012 & 7436.920 & $\underline{0.000}$ \\
\hline & Error & 0.000 & 12 & 0.000 & & \\
\hline & Total & 2.911 & 35 & & & \\
\hline \multirow{8}{*}{ WIP } & Efficiency & 14554.000 & 3 & 4851.000 & 10.780 & 0.001 \\
\hline & Inter-arrival & 13299.000 & 2 & 6649.000 & 14.770 & 0.001 \\
\hline & Workers & 7366.000 & 2 & 3683.000 & 8.180 & $\overline{0.006}$ \\
\hline & Efficiency*inter-arrival & 10108.000 & 6 & 1684.000 & 3.740 & 0.025 \\
\hline & Efficiency* Workers & 6047.000 & 6 & 1008.000 & 2.240 & $\overline{0.111}$ \\
\hline & Workers* inter-arrival & 7300.000 & 4 & 1825.000 & 4.050 & 0.026 \\
\hline & Error & 5401.000 & 12 & 450.100 & & \\
\hline & Total & 64075.000 & 35 & & & \\
\hline \multirow{8}{*}{ Workload AV } & Efficiency & 497.000 & 3 & 165.670 & 11.030 & 0.001 \\
\hline & Inter-arrival & 443.200 & 2 & 221.600 & 14.750 & $\overline{0.001}$ \\
\hline & Workers & 232.900 & 2 & 116.450 & 7.750 & $\underline{0.007}$ \\
\hline & Efficiency*inter-arrival & 312.300 & 6 & 52.050 & 3.460 & $\overline{0.032}$ \\
\hline & Efficiency* Workers & 196.200 & 6 & 32.700 & 2.180 & 0.118 \\
\hline & Workers* inter-arrival & 245.500 & 4 & 61.370 & 4.090 & $\underline{0.026}$ \\
\hline & Error & 180.300 & 12 & 15.020 & & \\
\hline & Total & 2107.400 & 35 & & & \\
\hline \multirow{8}{*}{ Workload DS } & Efficiency & 0.397 & 3 & 0.132 & 8.240 & $\underline{0.003}$ \\
\hline & Inter-arrival & 0.174 & 2 & 0.087 & 5.420 & 0.021 \\
\hline & Workers & 0.948 & 2 & 0.474 & 29.480 & 0.000 \\
\hline & Efficiency*inter-arrival & 0.091 & 6 & 0.015 & 0.950 & 0.497 \\
\hline & Efficiency* Workers & 0.079 & 6 & 0.013 & 0.820 & 0.573 \\
\hline & Workers* inter-arrival & 0.241 & 4 & 0.060 & 3.740 & $\underline{0.034}$ \\
\hline & Error & 0.193 & 12 & 0.016 & & \\
\hline & Total & 2.124 & 35 & & & \\
\hline
\end{tabular}


All main effects, except the efficiency for the throughput, and the majority of the two-way interactions were shown to be statistically significant at $\alpha=0.05$. Detailed results will be presented next in Section 5.1 before a discussion of the results is presented in Section 5.2

\subsection{Performance Assessment}

The main performance measures are presented in the form of performance curves. The curves present the percentage difference between the game theory and the benchmark for different settings in terms of the number of workers, the inter-arrival time, and the efficiency of the workers (see Fig. 1 and Fig. 2).
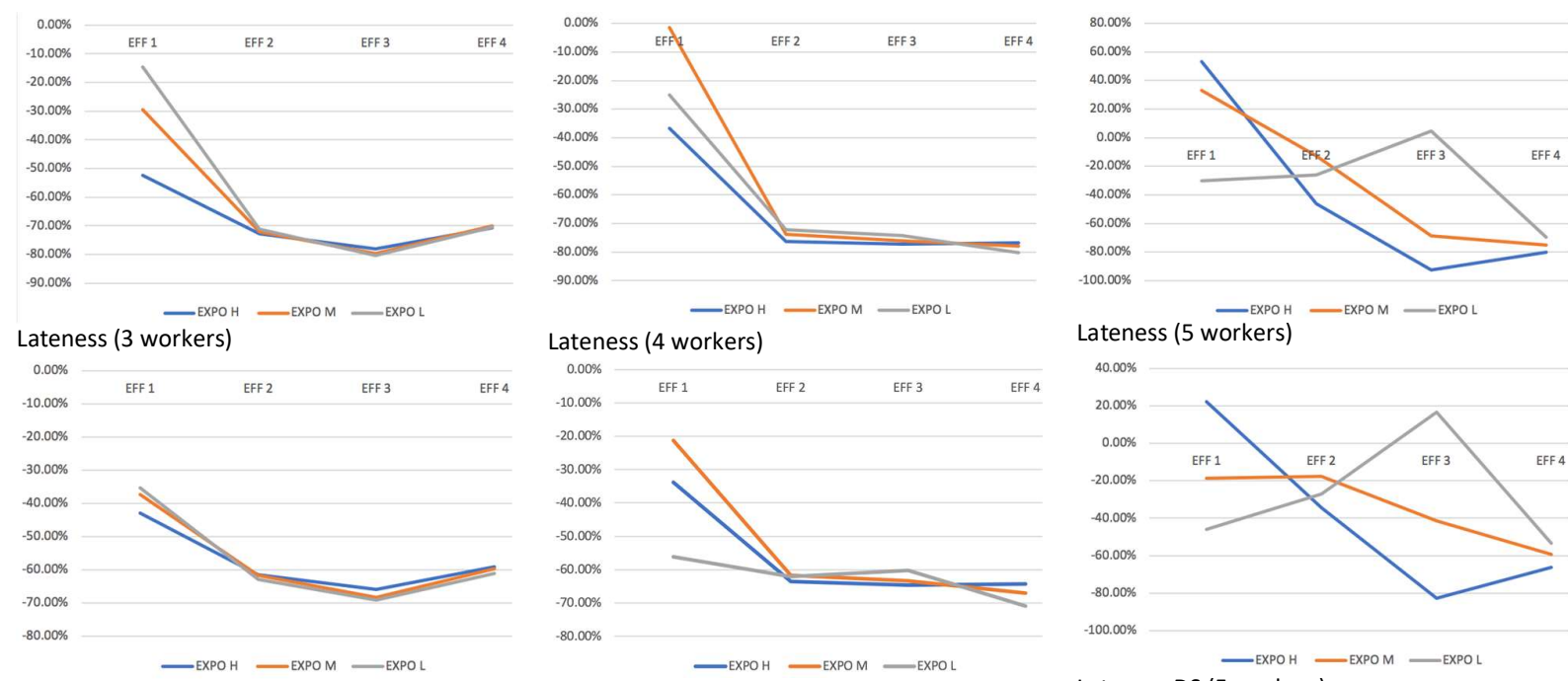

Lateness DS (3 workers)
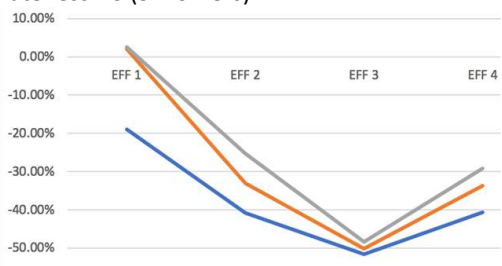

$-60.00 \%$

Tardy \% (3 workers)
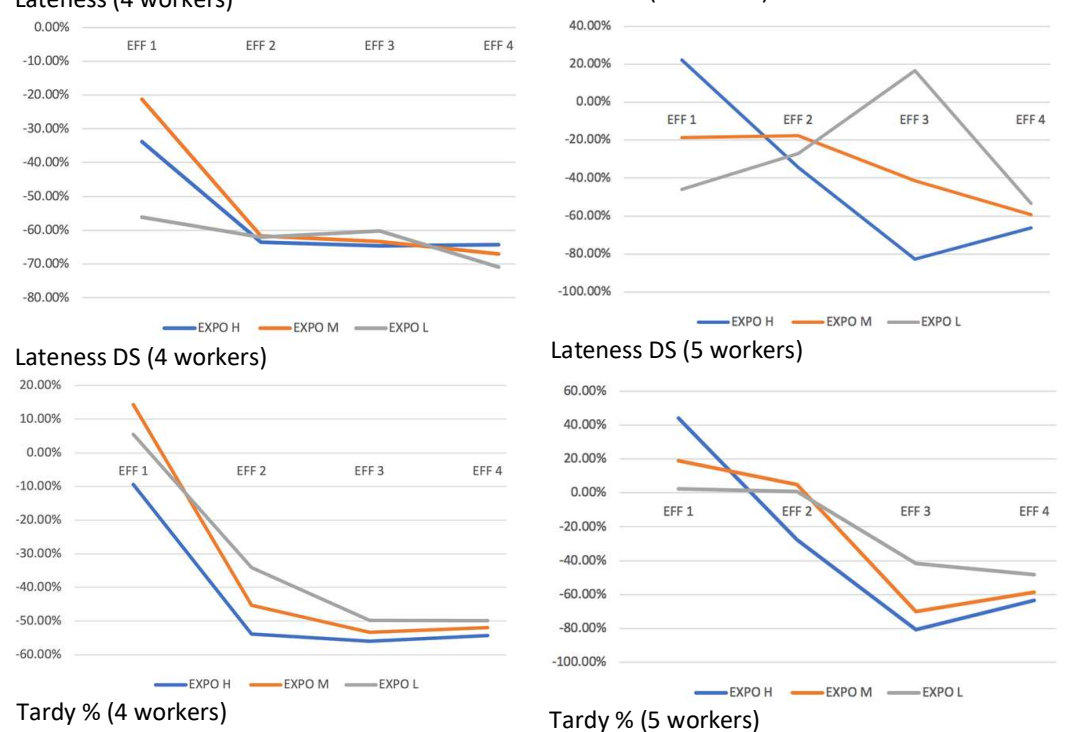

Fig. 1. Performance Assessment - Delivery Performance

The ability to deliver the job on time is significantly improved by our new approach to worker assignment. The average and the standard deviation of lateness (see Fig. 1) exhibit the same behavior. The game theory approach leads to a significant reduction in lateness. The reduction is higher when workers have a different efficiency level (EFF 2, 3 and 4). The inter-arrival parameter is of greater importance when the number of workers is 5 . In this case, each inter-arrival has a different trend on the lateness. The percentage of tardy jobs (Tardy \%, Fig. 1c) leads to the biggest improvement for the efficiency of EFF 3 of the workers; when the number of workers is 5, the improvements are relevant only when the efficiency of the workers is different. Meanwhile, in terms of the performance of the manufacturing system (see Fig. 2), the system time, WIP level, and throughput of the parts through the manufacturing system reduce with the proposed approach when the workers have different efficiencies. Meanwhile, the coefficient of variation for the workload distribution across the machines of the manufacturing system increases with the proposed approach. 

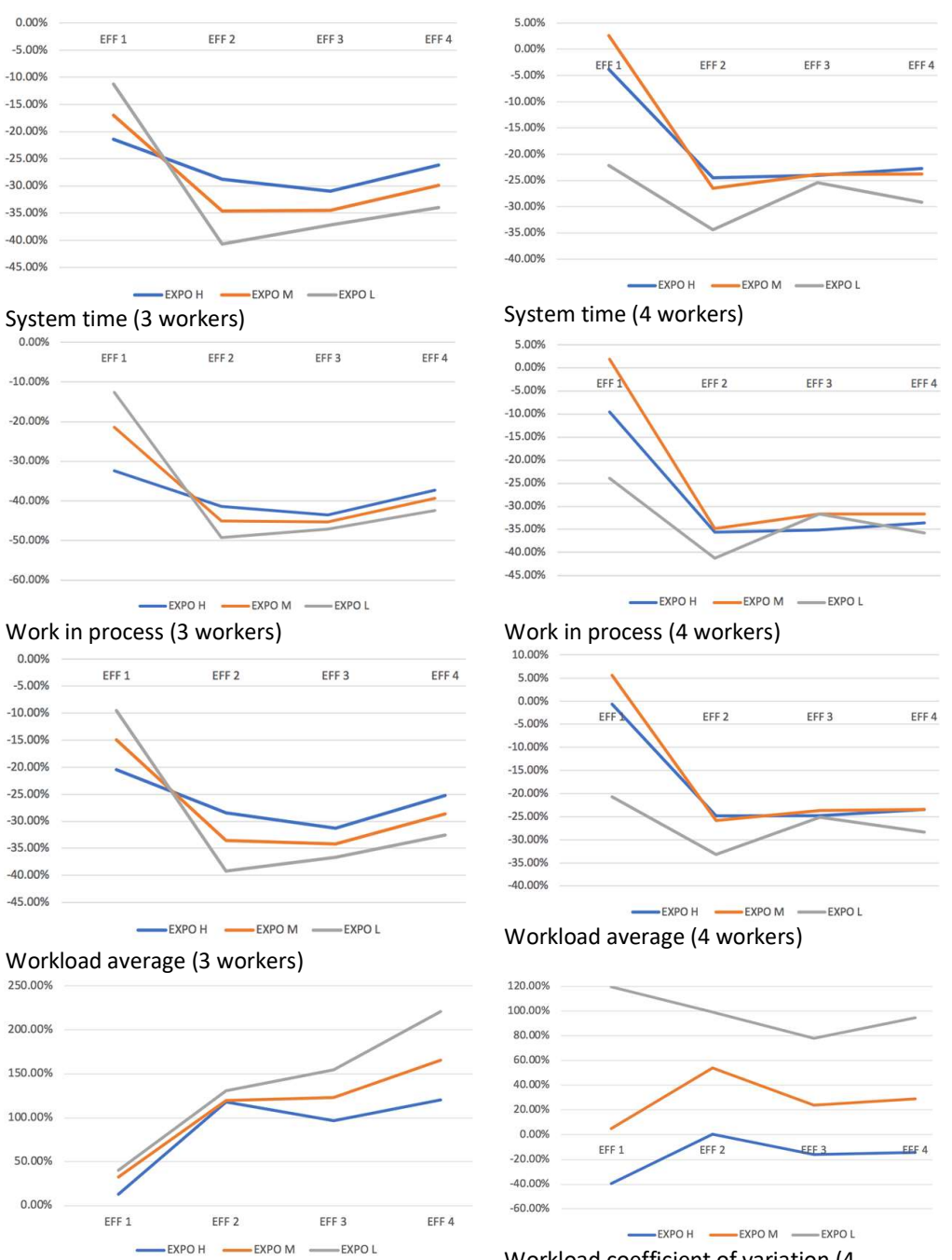

Workload coefficient of variation ( 3 workers)
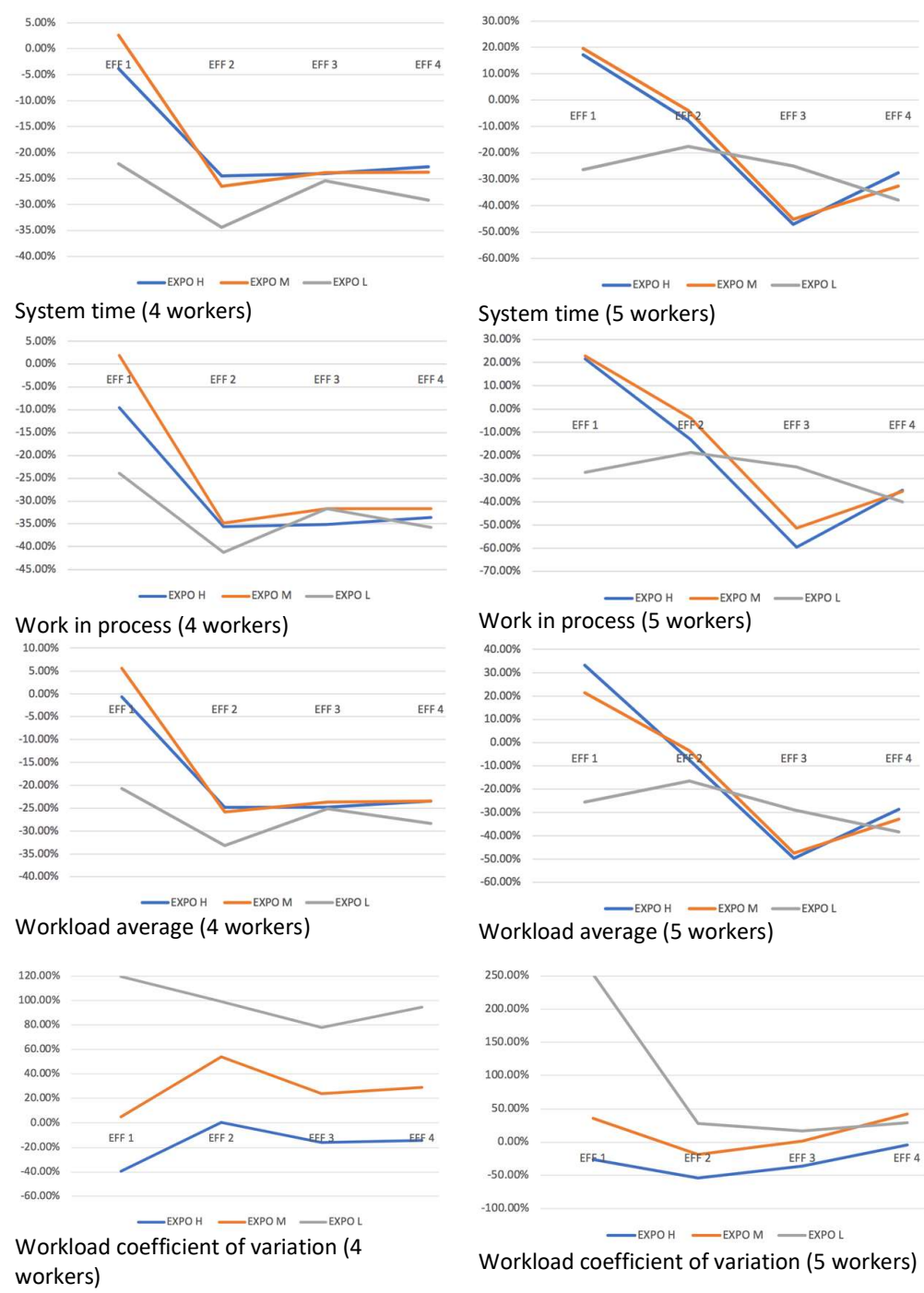

Fig. 2. Performance Assessment - Manufacturing System Performance

\subsection{Discussion of Results}

The proposed approach based on game theory improves all of the main performance measures analyzed; the different efficiencies of the workers is relevant for the game model. The game model is robust because all the conditions tested highlight better performances compared to the benchmark. When the number of workers increases, the different efficiencies is more important and has a major impact on the ability to obtain better results.

The parameters to set the game model are stable when the efficiency among the workers is significantly different. A major advantage of the proposed model is its periodic when rule, which allows for better planning in practice. In contrast, both a decentralized and centralized when rule may trigger a worker movement at any moment in time. However, it is important to determine the best value of $T p$ in order to obtain the improvement in performance shown. Table 4 reports the best value of $T p, \alpha$ and $\beta$ for each simulation class. The simulation experiments show the efficiency of the workers is relevant for these values. In particular, when the workers have a high difference of efficiency, the $T p$ tends to $5, \alpha$ to 1 and $\beta$ to 0 . Another important issue concerns the $T p$ value; the values of the $T p$ studied show that a low difference of $T p$ from the best value dramatically reduces the performance. Therefore, a when rule that leads to different values from the best $T p$ cannot be proposed in combination with the Gale-Shapley algorithm. 
Table 4

Best cases of Tp, $\alpha$ and $\beta$ values

\begin{tabular}{|c|c|c|c|}
\hline & \multicolumn{2}{|c|}{3 Workers } & \multirow[b]{2}{*}{ EXPO L } \\
\hline & EXPO H & EXPO M & \\
\hline EFF 1 & $\mathrm{Tp}=5 ; \alpha=0.8, \beta=0.2$ & $\mathrm{Tp}=10 ; \alpha=0.8, \beta=0.2$ & $T p=20 ; \alpha=0.9, \beta=0.1$ \\
\hline EFF 2 & $T p=5 ; \alpha=1, \beta=0$ & $T p=5 ; \alpha=1, \beta=0$ & $T p=5 ; \alpha=1, \beta=0$ \\
\hline EFF 3 & $T p=5 ; \alpha=1, \beta=0$ & $T p=5 ; \alpha=1, \beta=0$ & $T p=5 ; \alpha=1, \beta=0$ \\
\hline EFF 4 & $\mathrm{Tp}=5 ; \alpha=1, \beta=0$ & $\mathrm{Tp}=5 ; \alpha=1, \beta=0$ & $\mathrm{Tp}=5 ; \alpha=1, \beta=0$ \\
\hline \multicolumn{3}{|c|}{4 workers } & \\
\hline & EXPO H & EXPO M & EXPO L \\
\hline EFF 1 & $\mathrm{Tp}=5 ; \alpha=1, \beta=0$ & $\mathrm{Tp}=6 ; \alpha=1, \beta=0$ & $T p=10 ; \alpha=1, \beta=0$ \\
\hline EFF 2 & $T p=5 ; \alpha=1, \beta=0$ & $T p=5 ; \alpha=1, \beta=0$ & $T p=5 ; \alpha=1, \beta=0$ \\
\hline EFF 3 & $T p=5 ; \alpha=1, \beta=0$ & $T p=5 ; \alpha=1, \beta=0$ & $T p=5 ; \alpha=1, \beta=0$ \\
\hline EFF 4 & $\mathrm{Tp}=5 ; \alpha=1, \beta=0$ & $\mathrm{Tp}=5 ; \alpha=1, \beta=0$ & $\mathrm{Tp}=5 ; \alpha=1, \beta=0$ \\
\hline \multicolumn{3}{|c|}{5 workers } & \\
\hline & EXPO H & EXPO M & EXPO L \\
\hline EFF 1 & $\mathrm{Tp}=5 ; \alpha=1, \beta=0$ & $T p=15 ; \alpha=1, \beta=0$ & $T p=15 ; \alpha=1, \beta=0$ \\
\hline EFF 2 & $T p=5 ; \alpha=1, \beta=0$ & $\mathrm{~T} p=10 ; \alpha=1, \beta=0$ & $T p=15 ; \alpha=1, \beta=0$ \\
\hline EFF 3 & $\mathrm{~T} p=5 ; \alpha=1, \beta=0$ & $\mathrm{Tp}=5 ; \alpha=1, \beta=0$ & $T p=5 ; \alpha=1, \beta=0$ \\
\hline EFF 4 & $\mathrm{Tp}=5 ; \alpha=1, \beta=0$ & $\mathrm{Tp}=5 ; \alpha=1, \beta=0$ & $\mathrm{Tp}=5 ; \alpha=1, \beta=0$ \\
\hline
\end{tabular}

\section{Conclusions}

Manufacturing systems constrained by machine capacity and labor capacity are known as Dual Resource Constraint (DRC) shops. Although a broad literature on DRC shops exists, the application of game theory has not been investigated in this context. In response, our first research question asked: Can the GaleShapley Algorithm be adapted to the DRC job shop problem under different number of workers, workers' efficiency and inter-arrival time? Our second research question then asked: What is the performance improvement enabled by the Gale-Shapley Algorithm if compared to simple when and where rules? Using simulation, we have demonstrated how the Gale-Shapley algorithm has the potential to significantly improve DRC shop performance and that results are robust to changes in demand, the number of workers, and different efficiencies among the workers. The model proposed allows labor efficiency information to be included with a positive effect on performance when the efficiency among the workers is different. Moreover, the robustness of the approach is demonstrated by the improvements obtained for all performance measures considered and under different conditions of demand, the number of workers, and different efficiencies among the workers.

\subsection{Managerial Implications}

The proposed model can handle fluctuations in manufacturing systems due to labor turnover, absenteeism, different worker competences, etc. The model supports the assignment of workers to workstations following a periodic review policy. The game model reduces the number of worker movements compared to a centralized when rule that allows for worker re-allocation after each operation completion. While the game model may increase the number of transfers compared to a decentralized rule because it re-assigns all of the workers in the manufacturing system, it fixes the time when transfers occur. Hence, transfers are only triggered at fixed intervals (i.e. at a shift change or lunch break), whereas decentralized and centralized when rules may trigger a worker movement at any moment in time. The manager can use simulation with the game model to pursue a given performance target, adapting conditions such as the inter-arrival time to the number of workers available (absenteeism), or making capacity adjustments, such as the temporary introduction of a new machine. The game model assures a solution with low computational complexity is provided even when the number of workers and/or machines increase.

\subsection{Limitations and Future Research}

A limitation of our study is that we assume workers are fully interchangeable, and we omit behavioral factors such as learning and forgetting. The game model can include these features in the assignment problem and future research could therefore take these features into account. Meanwhile, the only performance measure that deteriorated in our study was the variation in the workload on the shop floor. 
Future research could therefore explore the interaction of the game model with workload control mechanisms to stabilize the workload.

\section{References}

Araz, O.U. (2005). A simulation based multi-criteria scheduling approach of dual resource constrained manufacturing systems with neural networks. Lecture Notes in Computer Science (including subseries Lecture Notes in Artificial Intelligence and Lecture Notes in Bioinformatics), 3809. LNAI, 1047- 1052.

Araz, O.U. (2007). Real-time controlled multi-objective scheduling through ANNs and fuzzy inference systems: The case of DRC manufacturing. Lecture Notes in Computer Science (including subseries Lecture Notes in Artificial Intelligence and Lecture Notes in Bioinformatics), 4490. LNCS (PART 4), 973-976.

Araz, Ö. U., \& Latif Salum, L. (2010) A multi-criteria adaptive control scheme based on neural networks and fuzzy inference for DRC manufacturing systems, International Journal of Production Research, 48(1), 251-270.

Bokhorst, J.A.C., Slomp, J., \& Gaalman, G.J.C. (2004). On the who-rule in dual resource constrained (DRC) manufacturing systems. International Journal of Production Research, 42(23), 5049-5074.

Buscemi, M.G., Montanari, U., \& Taneja, S. (2012). A game-theoretic analysis of grid job scheduling. Journal of Grid Computing, 10,501-519

Conway, R., Maxwell, W. L., \& Miller, L. W. (1967). Theory of Scheduling. Reading, MA: Addisson-Wesley.

Delgoshaei, A., Khairol, M., Ariffin, A., \& Ali, A. (2017). A multi- period scheduling method for trading-off between skilled-workers allocation and outsource service usage in dynamic CMS. International Journal of Production Research, 55(4), 997-1039.

Deming, L., \& Guo, X. (2014). Variable neighbourhood search for dual-resource constrained flexible job shop scheduling. International Journal of Production Research, 52(9), 2519-2529.

Opiyo, E. T., Ayienga, E., Getao, K., Okello-Odongo, W., Manderick, B., \& Nowé, A. (2008). Game theoretic multi-agent systems scheduler for parallel machines. International Journal of Computing and ICT Research, 1(1), 21-27.

ElMaraghy, H., Patel, V., \& Abdallah, I.B. (2000). Scheduling of manufacturing systems under dual-resource constraints using genetic algorithms. Journal of Manufacturing Systems, 19(3), 186-201.

Fredendall, L.D., Melnyk, S.A., \& Ragatz, G. (1996). Information and scheduling in a dual resource constrained job shop. International Journal of Production Research, 34(10), 2783-2802.

Gale, D., \& Shapley, L.S. (1962). College admissions and the stability of marriage. American Mathematical Monthly, 69, 1, 9-14.

Hamedi, M., Esmaieilian, G. R., Ismail, N., \& Ariffin, M.K.A. (2012). Capability-based virtual cellular manufacturing systems formation in dual-resource constrained settings using Tabu Search. Computers and Industrial Engineering, 62, 953-971.

Hopp, W.J., \& Van Oyen, M.P. (2004). Agile workforce evaluation: a framework for cross-training and coordination. IIE Transactions, 36(10), 919-940.

Kannan, V.R., \& Jensen, J.B. (2004). Learning and labour assignment in a dual resource constrained cellular shop. International Journal of Production Research, 42(7), 1455-1470,

Li, J., Huang, Y., \& Niu, X. (2016). A branch population genetic algorithm for dual-resource constrained job shop scheduling problem. Computers and Industrial Engineering, 102, 113-131.

Li, H., \& Lang, M. (2011). Research on dual-resource multi-objective flexible job shop scheduling

under uncertainty. In 2nd International Conference on Artificial Intelligence, Management

Science and Electronic Commerce (AIMSEC), Dengleng, China, 8-10 Aug. 2011.

Li, X., Gao, L., \& Li, W. (2012). Application of game theory based hybrid algorithm for multi-objective integrated process planning and scheduling. Expert Systems with Application, 39(1), 288-297.

Lenstra, J., \& Rinnooy Kan, A. (1979). Computational complexity of discrete optimization problems, in Discrete Optimization In Proceedings of the Advanced Research Institute on Discrete Optimization and Systems Applications of the Systems Science Panel of NATO and of the Discrete Optimization Symposium, Hammer, P., Johnson, E., and Korte, B. (eds), Elsevier, New York, NY, pp. 121-140.

Lobo, B.J., Wilson, J. R., Thoney, K.A., Hodgson, T. J., \& King, R. E. (2014). A practical method for evaluating worker allocations in large-scale dual resource constrained job shops. IIE Transactions, 46(11), 1209-1226. 
Lobo, B. J., Hodgson, T.J., King, R.E., Thoney, K.A., \& Wilson, J.R. (2013a). An Effective Lower Bound on Lmax in a Workerconstrained Job Shop. Computers and Operations Research, 40(1), 328-343.

Lobo, B., Hodgson, T., King, R., Thoney, K., \& Wilson, J. (2013b). Allocating job-shop manpower to minimize Lmax: optimality criteria, search heuristics, and probabilistic quality metrics. Computers \& Operations Research, 40(10), 2569-2584.

Melnyk, S., A., \& Ragatz, G., L. (1989) Order review/release: research issues and perspectives. International Journal of Production Research, 27(7),1081-1096

Mehravaran, Y., \& Logendran, R. (2013). Non-permutation flowshop scheduling with dual resources. Expert Systems with Applications, 40(13), 5061-5076.

Nembhard, D.A., ed. (2007). Workforce cross training. Boca Raton, FL: CRC Press, Taylor \& Francis Group.

Opiyo, E., T., O., Ayienga, E., Getao, E., K., Okello-Odongo, W., Manderick, B., Nowé, A. (2008). Game theoretic multi-agent systems scheduler for parallel machines. International Journal of Computing and ICT Research, 1(1), 21-27.

Oosterman, B., Land, M., \& Gaalman, G. (2000). The influence of shop characteristics on workload control. International Journal of Production Economics, 68(1), 107-119.

Patel, V. (1997). Scheduling in A Dual Resource Constrained System Using Genetic Algoritms. (Windsor: University of Windsor).

Renna, P. (2017). Decision-making method of reconfigurable manufacturing systems' reconfiguration by a Gale-Shapley model. Journal of Manufacturing Systems, 45, 149-158.

Renna P. 2015. Workload control policies under continuous order release. Production Engineering, 9, 5-6, $655-664$.

Salum, L., \& Araz Ö. U. (2009). Using the when/where rules in dual resource constrained systems for a hybrid push-pull control. International Journal of Production Research, 47(6), 1661-1677.

Sammarco, N., Fruggiero, F, Neumann, W.P., \& Lambiase, A. (2014). Agent-based modelling of movement rules in DRC systems for volume flexibility: human factors and technical performance. International Journal of Production Research, 52(3), 633-650.

Satoglu, S. I., \& Suresh, N.C. (2009). A goal-programming approach for design of hybrid cellular manufacturing systems in dual resource constrained environments. Computers and Industrial Engineering, $56,560-575$.

Sun, D., He, W., Zheng, L., \& Liao, X. (2014). Scheduling flexible job shop problem subject to machine breakdown with game theory. International Journal of Production Research, 52(13), 3858-3876.

Teo, C-P., Sethuraman, J., \& Tan W.-P. (2001). Gale-Shapley stable marriage problem revisited: Strategic issues and applications. Management Science, 47(9),1252- 1267.

Thürer, M., Stevenson, M., \& Renna, P. (2019). Workload control in dual-resource constrained high-variety shops: an assessment by simulation. International Journal of Production Research, 57(3), 931-947.

Thürer, M., Silva, C., \& Stevenson, M. (2014a). Controlled order release: a performance assessment in job shops with sequence dependent set-up times. Production Planning \& Control, 25(7), 603-615.

Thürer, M., Qu, T., Stevenson, M., Maschek, T., \& Godinho Filho, M. (2014b). Continuous Workload Control Order Release Revisited: An Assessment by Simulation. International Journal of Production Research, 52(22), 6664-6680

Zhang, J., Wang, W., Xu, X. (2017). A hybrid discrete particle swarm optimization for dual-resource constrained job shop scheduling with resource flexibility. Journal of intelligent Manufacturing, 28, 19611972.

Zheng, X.-1., \& Wang, L. (2016). A knowledge-guided fruit fly optimization algorithm for dual resource constrained flexible job-shop scheduling problem, International Journal of Production Research, 54(18), $5554-5566$

Xu, J., Xu, X., \& Xie, S.Q. (2011). Recent developments in Dual Resource Constrained (DRC) system research. European Journal of Operational Research, 215, 309-318.

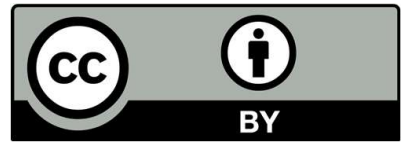

(C) 2020 by the authors; licensee Growing Science, Canada. This is an open access article distributed under the terms and conditions of the Creative Commons Attribution (CCBY) license (http://creativecommons.org/licenses/by/4.0/). 